\title{
Clinical Outcome of Acute Ischemic Strokes in Patients with COVID-19
}

\author{
Julie Calmettes ${ }^{a}$ Roxane Peres ${ }^{b}$ Bruno Goncalves ${ }^{c, d}$ David Varlan ${ }^{a}$ \\ Guillaume Turc ${ }^{c}$ Michael Obadiab Clotilde Nardin ${ }^{a} \quad$ Elodie Meppiel ${ }^{a}$ \\ Thomas De Broucker ${ }^{a} \quad$ Mikael Mazighi ${ }^{e} \quad$ Aicha Lyoubia
}

${ }^{a}$ Neurology Department, Delafontaine Hospital, Saint Denis, France; ${ }^{b}$ Neurology Department, Fondation Adolphe de Rothschild, Paris, France; ' CNeurology Department, GHU Paris Psychiatry and Neuroscience, Paris, France; ${ }^{\text {Paulo }}$ Niemeyer State Brain Institute, Intensive Care Unit, Rio de Janeiro, Brazil; eNeuroradiology Department, Fondation Adolphe de Rothschild, Paris, France

\section{Keywords}

Acute ischemic stroke · COVID-19 - Outcome

\begin{abstract}
Introduction: Acute ischemic stroke (AIS) and thrombotic events (TEs) were reported in patients with COVID-19. Clinical outcome of AIS in the course of COVID-19 remains unknown. We compared early clinical outcome and mortality of COVID-positive (+) patients admitted for AIS with COVIDnegative (-) ones. We hypothesized that COVID+ patients would have poorer clinical outcomes and present a higher rate of TEs and mortality compared with COVID- ones. Methods: In this multicentric observational retrospective study, we enrolled patients over 18 years old admitted for AIS in 3 stroke units of the Parisian region during lockdown from March 17, 2020, to May 2, 2020. COVID-19 status as well as demographic, clinical, biological, and imaging data was collected retrospectively from medical records. Poor outcome was defined as modified Rankin score (mRS) 3-6 (3-6) at discharge. We also compared TE frequency and mortality rate through a composite criterion in both groups. Results: Two hundred and sixteen patients were enrolled; mean age was 68 years old, and $63 \%$ were male. Forty patients were CO-
\end{abstract}

karger@karger.com

(c) 2021 S. Karger AG, Basel

www.karger.com/ced

Karger!
VID+ (18.5\%) and 176 were COVID-. Obesity was statistically more frequent in the COVID+ group ( $36 \mathrm{vs.} 13 \% p<0.01$ ). The percentage of patients with mRS (3-6) at discharge was higher in the COVID+ group compared with the COVIDgroup ( 60 vs. $41 \%, p=0.034$ ). The main predictor of presenting a mRS (3-6) at discharge was high NIHSS score at admission (OR, Cl 95\%: 1.325, 1.22-1.43). Mortality rate was higher in the COVID+ group (12 vs. $3.4 \%, p=0.033$ ) as well as TE frequency (15 vs. $2.8 \%, p<0.01)$. Conclusion: In this study, patients with AIS infected by SARS-CoV-2 showed a poorer early outcome than COVID- ones. However, when compared to other factors, COVID-19 was not a significant predictor of poor outcome. Vascular morbidity and mortality rates were significantly higher in the COVID+ group compared with the COVID- group.

(c) 2021 S. Karger AG, Basel

\section{Introduction}

Acute ischemic strokes (AISs) were reported during the course of COVID-19, representing sometimes the initial clinical manifestation of the disease [1]. In those reports, prevalence of AIS during COVID-19 varies be- 
tween 1.6 and $6.3 \%$ [ $1-3$ ], and it represents $23.4 \%$ of neurological manifestations of the disease [4]. Case reports suggested that COVID-19 can affect younger patients than usual AIS population $[3,5]$. Results of an American cohort study showed that AIS patients infected by SARSCoV-2 had higher National Institute Health Stroke Scale (NIHSS) scores and D-dimer levels at admission compared with non-infected ones [6].

There is significant evidence that in addition to the respiratory burden, COVID-19 is associated with an unexpected rate of thrombotic events (TEs) varying from $7.7 \%$ in in-hospital patients to $31 \%$ in critically ill patients (7). Venous TEs were the most frequent thrombotic complications leaded by pulmonary embolism (up to 81\%) [7]. This coagulopathy may represent a severity marker of the disease as it was more frequently observed in non-survivor COVID-19 patients (50 vs. 7\%) [8].

To date, no study evaluated the prognosis of AIS patients infected by SARS-CoV-2. In this context, we aimed to compare the clinical prognosis of COVID-positive (COVID+) patients with COVID-negative (COVID-) ones in a population of AIS patients admitted in 3 stroke units of the Parisian region during the French lockdown. We also compared the occurrence of TE and mortality in both populations.

\section{Methods}

\section{Study Design and Patients}

We conducted a multicentric retrospective study in 3 stroke units of the Parisian region (Delafontaine Hospital, Adolphe de Rothschild Foundation Hospital, and Sainte Anne Hospital) during lockdown. We enrolled all patients over 18 years admitted for AIS in these hospitals from March 17, 2020, where French lockdown was declared, to May 2, 2020.

Thoracic CT scan and nasal SARS-CoV-2 PCR were performed in routine care, at the discretion of referral clinicians. Patients were considered as infected by SARS-CoV-2 (COVID+) if either nasal SARS-CoV-2 PCR was positive or COVID-19 aspect was described on thoracic CT scan. To exclude potential nosocomial COVID-19, patients presenting with symptoms and for whom diagnosis was made $48 \mathrm{~h}$ or more after admission were excluded from the analysis, following the definition of nosocomial infection. Patients were considered as COVID - if they had no COVID-19 evidence on thoracic CT-scan and/or negative SARS-CoV-2 PCR. When SARSCoV-2 PCR and thoracic CT scan were not performed, patients were considered COVID-.

\section{Data Collection}

Demographic and clinical data at admission were collected retrospectively from medical records in the 3 stroke units by vascular neurologists. In accordance with French legislation, each patient was informed of his/her participation in this study and was offered the possibility to withdraw. The study was compliant to the Commission Nationale de l'Informatique et des Libertés (CNIL, reference number 2217894 v0) and to the Ethics Committee requirements (reference number 20.05.05.49236).

Following laboratory results were collected at admission: leukocyte count, platelet count, C-reactive protein (CRP) level, prothrombin time, and activated thromboplastin time. Stroke severity at admission was assessed prospectively by using the NIHSS [9]. Stroke subtype was classified based on the Trial of ORG 10172 in Acute Stroke Treatment (TOAST) classification [10]. Stroke territory and revascularization procedures were gathered. The modified Rankin score (mRS) [11] at discharge (or last 1 available when patient still in charge) as well as inhospital TE and death was also collected. All clinical scores gathered were prospectively assessed by referral clinicians in routine clinical practice.

\section{Study Outcomes}

The primary outcome of this study was poor outcome at discharge, defined as mRS (3-6). Secondary outcome was defined as a composite criterion including in-hospital TE and death. TEs were defined as deep vein thrombosis, pulmonary embolism, myocardial infarction, and peripheral artery occlusion.

\section{Statistical Analysis}

Statistical analysis was performed with R software. Differences between COVID+ and COVID- population for covariates and clinical outcome were assessed using $\chi^{2}$ analysis, $t$ test, or nonparametric test according to distribution. In addition, multivariate analysis (mixed model) was performed to evaluate the covariate weight of AIS severity status including COVID status, age, diabetes, and initial NIHSS score.

\section{Results}

\section{Baseline Characteristics}

Two hundred twenty patients were admitted for AIS in one of the 3 stroke units between March 17 and May 2 , 2020. Three were excluded for missing key data in medical records. One was excluded because COVID-19 diagnosis was made $>1$ week after admission making COVID-19 status unclear. Two hundred and sixteen patients were included in the study (Table 1). Forty patients were diagnosed with COVID-19 (18.5\%), 26 had positive SARS-CoV-2 PCR; thoracic CT scan was performed in 35 patients and showed COVID evidence in 32 (80\%) patients. One hundred seventy-six patients were considered as COVID-. In the COVID- group, SARS-CoV-2 PCR was performed in 38 patients $(21.5 \%)$ and was negative in all of them. Thoracic CT scan was performed in $49(28 \%)$ cases and showed no evidence of COVID-19 in all cases.

Baseline characteristics are summarized in Table 1. Mean age did not differ significantly between the 2 groups 
Fig. 1. Distribution of $\mathrm{mRS}$ at discharge in both groups. mRS, modified Rankin Score; COVID-, COVID-negative; COVID+, COVID-positive.



Table 1. Baseline characteristics of patients

\begin{tabular}{|c|c|c|c|c|}
\hline & $\begin{array}{l}\text { COVID- } \\
(n=176)\end{array}$ & $\begin{array}{l}\text { COVID+ } \\
(n=40)\end{array}$ & $p$ value & Test \\
\hline Sex, men, $n(\%)$ & $111(63)$ & $25(62)$ & 0.95 & $\chi^{2}$ \\
\hline Age, mean (SD) & $68.1( \pm 14.4)$ & $64.8( \pm 13.5)$ & 0.17 & Welch \\
\hline \multicolumn{5}{|l|}{ Medical history, $n(\%)$} \\
\hline Diabetes mellitus & $42(24)$ & $12(30)$ & 0.42 & $\chi^{2}$ \\
\hline Hyperlipidemia & $57(32)$ & $14(35)$ & 0.75 & $x^{2}$ \\
\hline Atrial fibrillation & $23(13)$ & $6(15)$ & 0.75 & $x^{2}$ \\
\hline Hypertension & $101(58)$ & $22(58)$ & 0.98 & $x^{2}$ \\
\hline Obesity & $17(13)$ & $9(36)$ & $<0.01$ & Fischer \\
\hline History of TE & $24(14)$ & $6(15)$ & 0.82 & $\chi^{2}$ \\
\hline History of stroke or TIA & $31(18)$ & $8(20)$ & 0.72 & $x^{2}$ \\
\hline \multicolumn{5}{|l|}{ Treatment at admission, $n(\%)$} \\
\hline Antiplatelet therapy & $60(34)$ & $11(28)$ & 0.42 & $\chi^{2}$ \\
\hline Anticoagulant & $17(9.7)$ & $6(15)$ & 0.39 & Fischer \\
\hline ARA-2 & $42(24)$ & $4(10)$ & 0.053 & $\chi^{2}$ \\
\hline ACE inhibitors & $23(13)$ & $12(30)$ & $<0.01$ & $x^{2}$ \\
\hline \multicolumn{5}{|l|}{ Laboratory results } \\
\hline $\mathrm{CRP}$, mean (range), mg/L & $20.1(0-362)$ & $51.6(1-263)$ & $<0.01$ & Welch \\
\hline Leukocytes, mean (range), g/L & $8,798(1,265-23,900)$ & $9,640(3,000-37,380)$ & 0.35 & Welch \\
\hline Platelets, mean (range), g/L & $251(98-494)$ & $358(83-755)$ & $<0.001$ & Welch \\
\hline APTT, mean (range) & $1.06(0.78-1.88)$ & $1.07(0.60-2.09)$ & 0.81 & Welch \\
\hline $\mathrm{PT}$, mean (range), \% & $87.4(18-116)$ & $84.8(42-124)$ & 0.38 & Welch \\
\hline \multicolumn{5}{|l|}{ Initial stroke severity } \\
\hline NIHSS score at admission, mean (SD) & $7.14( \pm 7.62)$ & $10.2( \pm 9.72)$ & 0.071 & Welch \\
\hline
\end{tabular}

TIA, transient ischemic attack; ARA-2, angiotensin II receptor antagonists; ACE, angiotensin-converting enzyme; CRP, C-reactive protein; APTT, activated partial thromboplastin time; PT, prothrombin time; NIHSS: National Institutes of Health Stroke Scale; TE, thrombotic event; COVID-, COVID-negative; COVID+, COVIDpositive. 
Fig. 2. Multiple ischemic lesions pattern in 3 COVID+ patients. Diffusion-weighted 1.5 T MRI. See arrows: hyperintensities in different arterial territories in 3 patients with COVID-19.

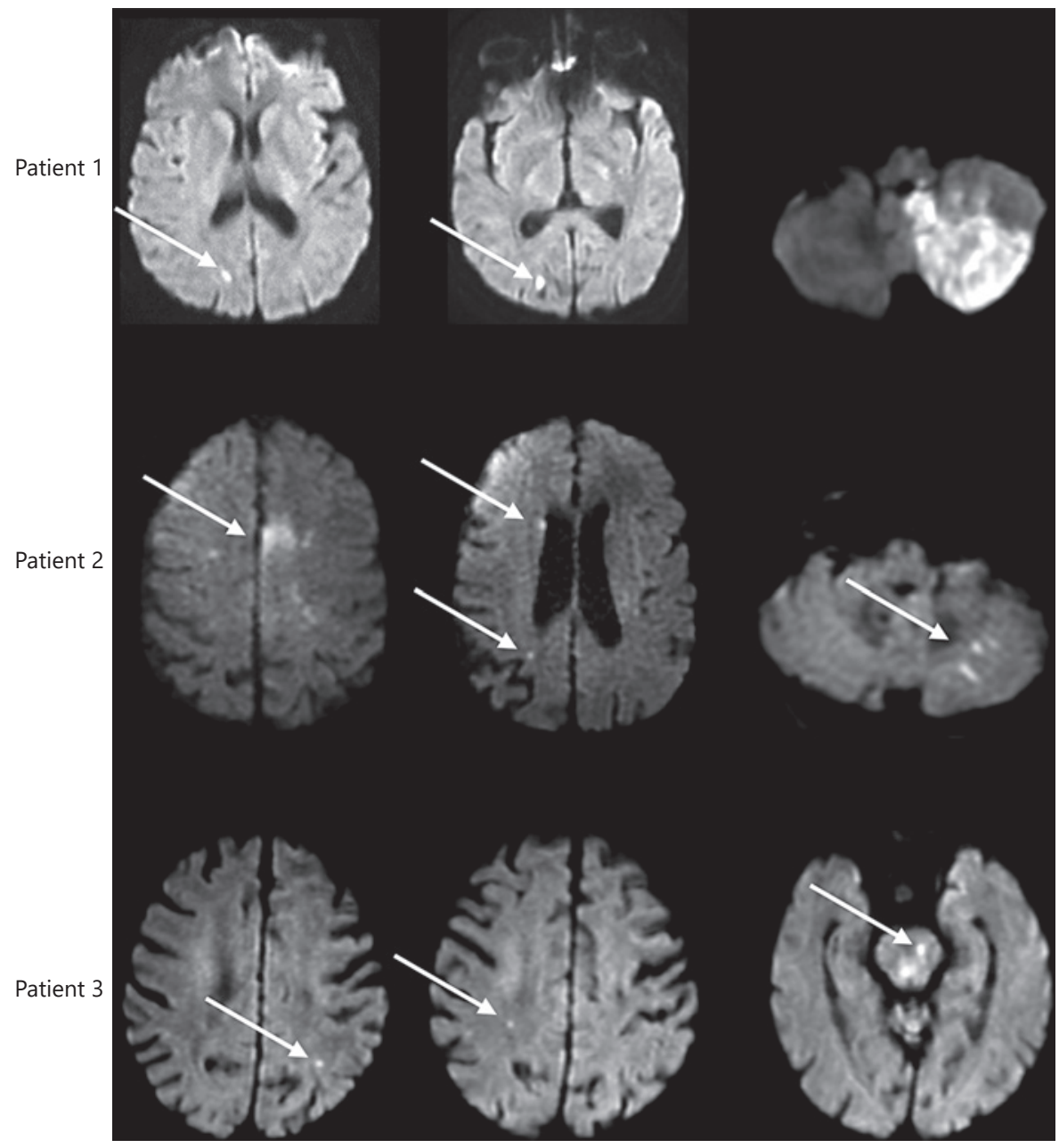

with, respectively, $68.1( \pm 14.4)$ in the COVID- group and $64.8( \pm 13.5)$ in the COVID+ group with a majority of male sex in both groups (63\% in COVID- and $62 \%$ in COVID+, $p=0.95$ ). Obesity, defined as BMI $\geq 30 \mathrm{~kg} / \mathrm{m}^{2}$, was significantly more frequent in the COVID+ group than in the COVID-one ( 13 vs. $36 \%, p<0.01)$. There was no significant difference in treatment at admission except for converting enzyme inhibitors (CEIs) with $30 \%$ in the COVID+ group and $13 \%$ in the COVID- group $(p<$ 0.01 ). CRP level and platelet count were significantly more elevated in the COVID+ group, respectively, 51.6 versus $20.1 \mathrm{mg} / \mathrm{L}(p<0.01)$ and 358 versus $251 \mathrm{~g} / \mathrm{L}(p<$ $0.001)$. NIHSS score at admission was higher in the COVID+ group with a mean score of $10.2( \pm 9.72)$ and 7.14 $( \pm 7.62)$ in the COVID- group, but no significance was reached $(p=0.071)$.

\section{Stroke Characteristics and Revascularization}

Procedures

For COVID+ patients, the median time from first COVID-19 symptom was 12 days (0-46 days). Stroke was the initial clinical manifestation in 12 patients (30\%). Regarding stroke territory, there was a trend for a predominance of multiple (Fig. 2) (26 vs. $14 \% ; p=0.089)$ and cerebral anterior strokes ( 23 vs. $10 \% ; p=0.058$ ) in the COVID+ group (Table 2). There was no difference in stroke mechanisms between 2 groups. Alteplase (22 vs. $14 \%, p=0.11$ ) and mechanical thrombectomy (28 vs. $16 \%, p=0.16$ ) were more often performed in the COVID+ group, but this difference was not statistically significant.

\section{Study Outcomes}

Primary and secondary outcomes are summarized in Table 3 . There were significantly more patients with $\mathrm{mRS}$ 
Table 2. Territories, mechanism, and revascularization of ischemic strokes

\begin{tabular}{|c|c|c|c|c|}
\hline & $\begin{array}{l}\text { COVID- } \\
(n=176)\end{array}$ & $\begin{array}{l}\text { COVID+ } \\
(n=40)\end{array}$ & $p$ value & Test \\
\hline \multicolumn{5}{|l|}{ Stroke territories, $n(\%)$} \\
\hline Anterior cerebral artery, $n$ & $18(10)$ & $9(23)$ & 0.058 & Fischer \\
\hline Middle cerebral artery, $n$ & $109(63)$ & $20(51)$ & 0.18 & $x^{2}$ \\
\hline Posterior cerebral artery, $n$ & $25(14)$ & $7(18)$ & 0.58 & $\chi^{2}$ \\
\hline Basilar artery, $n$ & $28(16)$ & $6(15)$ & 0.9 & $\chi^{2}$ \\
\hline Multiple territory, $n$ & $25(14)$ & $10(26)$ & 0.089 & $x^{2}$ \\
\hline \multicolumn{5}{|l|}{ Stroke mechanism, $n(\%)$} \\
\hline Large-artery atherosclerosis, $n$ & $44(26)$ & $9(22)$ & 0.69 & $\chi^{2}$ \\
\hline Cardioembolism, $n$ & $56(33)$ & $10(25)$ & 0.33 & $x^{2}$ \\
\hline Small-vessel occlusion, $n$ & $13(7.6)$ & $1(2.5)$ & 0.48 & Fischer \\
\hline Other, $n$ & $5(2.9)$ & $3(7.5)$ & 0.18 & Fischer \\
\hline Undetermined, $n$ & $61(35)$ & $18(45)$ & 0.26 & $x^{2}$ \\
\hline \multicolumn{5}{|l|}{ Revascularization procedures, $n(\%)$} \\
\hline Thrombectomy, $n$ & $29(16)$ & $11(28)$ & 0.11 & $x^{2}$ \\
\hline Alteplase, $n$ & $24(14)$ & $9(22)$ & 0.16 & $x^{2}$ \\
\hline
\end{tabular}

Table 3. Primary and secondary outcomes

\begin{tabular}{|c|c|c|c|c|}
\hline & $\begin{array}{l}\text { COVID- } \\
(n=176)\end{array}$ & $\begin{array}{l}\text { COVID+ } \\
(n=40)\end{array}$ & $p$ value & Test \\
\hline \multicolumn{5}{|l|}{ Primary outcome } \\
\hline $3-\mathrm{mRS}-6, n(\%)$ & $73(41)$ & $24(60)$ & 0.034 & $\chi^{2}$ \\
\hline \multicolumn{5}{|l|}{ Secondary outcome } \\
\hline Morbimortality composite criterion, (CI 95\%) & $0.06(0.03-0.10)$ & $0.25(0.14-0.40)$ & $<0.01$ & Fischer \\
\hline OR (CI 95\%) & & $5.0(1.95-12.80)$ & 0.01 & \\
\hline TE, $n(\%)$ & $5(2.8)$ & $6(15)$ & $<0.01$ & Fischer \\
\hline In-hospital death, $n(\%)$ & $6(3.4)$ & $5(12)$ & 0.033 & Fischer \\
\hline
\end{tabular}

mRS, modified Rankin Scale; CI, confidence interval; TE, thrombotic event.

Table 4. Logistic regression model on unfavorable mRS at discharge

\begin{tabular}{lllr}
\hline & Odds ratio & CI 95\% & $p$ value \\
\hline Age & 1.034 & $1.005-1.063$ & 0.019 \\
Diabetes mellitus & 1,005 & $0.433-2.335$ & 0.990 \\
NIHSS at admission & 1.325 & $1.222-1.438$ & $<0.001$ \\
COVID-19 & 1.923 & $0.759-4.876$ & 0.168 \\
\hline
\end{tabular}

NIHSS, National Institutes of Health Stroke Scale.
(3-6) at discharge in the COVID+ group (shown in Fig. 1) compared with the COVID- group (60 vs. $40 \%, p=$ $0.034)$. When adjusted to age, diabetes, and initial NIHSS scores, COVID+ status was associated with poor outcome (Table 4); this association did not reach significance (OR, CI 95\%: 1.923, 0.75-4.8).

TE and mortality composite criteria were higher in the COVID+ group than in the COVID- group (25 vs. $6 \%) ; p<0.01$. Death in the COVID+ group was consecutive to malignant infarction in 3 cases and respiratory failure in 2 others. Six TEs were observed in the COVID+ group: 3 peripheral artery occlusions, 1 myocardial infarction, 1 deep vein thrombosis, and 1 pulmonary embolism. 


\section{Discussion}

In a sample of $>200$ patients admitted for AIS, $18.5 \%$ were COVID+. Frequency of COVID+ in series of all coming AIS and during the same period is not clearly assessed. Jillella et al. [12] reported a frequency of $2.5 \%$ of COVID+ cases in their series of AIS. However, the study period was very different from ours and therefore cannot be correctly compared. We observed an overrepresentation of poor outcome in patients suffering from AIS concomitantly with SARS-CoV-2 infection. Mortality rate was higher in the COVID+ group (12 vs. $3.4 \%, p=0.033$ ), as well as TE frequency ( 15 vs. $2.8 \%, p<0.01$ ). The mortality rate combined with TE rate $(25 \%)$ was higher in the COVID+ group. The strongest predictor of poor outcome was the NIHSS score at admission.

Though clinical severity at admission was reported to be high in COVID+ patients suffering from AIS (6), no data are available in respect to prognosis and clinical outcome of these patients. The 30-day case fatality in France for AIS reached $10.7 \%$ in 2015 [13] with in-hospital mortality of $9.4 \%$ in 2014 [14]. In our study, in-hospital mortality rate of COVID+ patients was of $12 \%$ versus $3.4 \%$ in COVID- ones. Of note, mortality was not only driven by respiratory failure in the COVID+ group but also by malignant infarction.

These findings suggest that, in addition to an enhanced severity at admission, patients presenting concomitantly with AIS and SARS-CoV-2 infection present a higher risk of early unfavorable outcome and death than usually observed in general AIS population as well as in contemporary COVID-controls. Together with the higher level of TE observed, this highlights the importance of vascular comorbidity as part of the severity spectrum of COVID-19. As the median time delay from first COVID-19 symptom to stroke was 12 days and given the severity of this population prognosis, monitoring should be undertaken for these patients during this time period.

As described, we also found that obesity was overrepresented in severe COVID-19 patients and that the most frequently observed comorbidity was arterial hypertension $[1,7]$. The frequency of these 2 vascular risk factors in severe forms of COVID-19 as well as in AIS patients do suggest that vascular comorbidity can represent a severity predictor in this affection. Endothelial dysfunction in COVID-19 disease as shown in histopathology studies [15] also supports this hypothesis.

Interestingly, in our study, patients with AIS and COVID were more often treated with angiotensin-convert- ing enzyme (ACE) inhibitors (30 vs. $13 \%, p<0.01)$. In vitro and in vivo studies $[15,16]$ highlighted the key role played by ACE2 in COVID-19 as functional receptor for SARS-CoV-2. However, the role played by ACE2 in this disease remains unclear; basic research studies displayed a potential enhanced disease severity caused by overexpression of ACE2 with a paradox lung protecting role in mice infected with SARS-CoV-2 [16]. Thus, further studies are needed to provide clear guidelines about CEI use in COVID-19.

Regarding thrombosis and inflammation markers, CRP level and platelet count were higher in the COVID+ group. This is quite expected as part of the inflammatory status of the infection. However, in the Chinese COVID-19 in-patient population, when compared to survivors, non-survivors were more likely to present a coagulopathy state (50 vs. 7\%) [8]. The inflammatory status of the infection seems to be 1 of the important biological features of the patients presenting with AIS and COVID-19. CRP and platelet count as well as D-dimer levels, as suggested by a recent similar study [6] may be part of the evaluation of severe disease form in patients infected with SARS-CoV-2. Thus, identifying routine biological markers predictive of severe vascular forms may be of important clinical interest to maximize vascular prevention strategy in SARS-CoV-2-positive patients.

Acute infection and more particularly influenza is a well-recognized risk enhancer for cardiovascular mortality [17] and AIS [18]. We and others [6] observed a trend towards an over-representation of AIS of undetermined cause in COVID+ patients. Acute viral infection can therefore be an underestimated etiology for AIS of undetermined source or a trigger for AIS of other cause in clinical routine.

Our study has several limitations, first of which is the retrospective nature of the design. Moreover, when adjusted to other potential confounding factors, the association between COVID+ status and poor clinical outcome did not remain statistically significant. The presence of large vessel occlusion has been reported to be frequent in COVID+ patients $[19,20]$. In our study, there was a trend towards more thrombectomy in this group but large vessel occlusion rate was not assessed in our study. This, in addition to other confounding factors such as delays for admission or infarct sizes, has not been assessed and can therefore represent potential bias for the results. Besides, we only gathered information about patients admitted in stroke units and not in critical care units, which represents a selection bias and may underestimate severe forms of AIS or AIS incidence in critically ill patients with COV- 
ID-19. Regarding COVID-19 status, an important part of COVID- patients were not tested for SARS-CoV-2, and thus COVID status remains uncertain, as asymptomatic forms of SARS-CoV-2 have been described. We also did not collect information about respiratory status of COVID+ patients, which may have underestimated its role in poor outcome. Besides, only hospitalized patients were involved; therefore, we cannot generalize our findings to ambulatory and general population. Further studies are needed to evaluate the proper risk of this population to develop severe COVID-19 form to insure a personalized management of patients, especially before applying invasive prevention or therapeutic measures.

\section{Conclusion}

Patients with AIS and COVID-19 showed a high rate of mortality and TE. When compared to other factors such as age or NIHSS scores, COVID-19 was not a statistically significant predictor of poor outcomes in our AIS population. Obesity and inflammatory biologic status were more frequently observed in COVID+ patients. A special interest should be provided to these clinical and biological features in COVID+ patients to prevent vascular complications that seem to be part of the disease severity spectrum. Further studies are needed to identify strong predictors of severe vascular forms of COVID-19 in the general population.

\section{Statement of Ethics}

In accordance with French legislation, each patient was informed of his/her participation in this study and was offered the possibility to withdraw. The study was compliant to the Commission Nationale de l'Informatique et des Libertés (CNIL, reference number 2217894 v0) and to Ethics Committee requirements (reference number 20.05.05.49236).

\section{Conflict of Interest Statement}

No conflict of interest to declare.

\section{Funding Sources}

Dr. Bruno Gonçalves: Coordenação de Aperfeiçoamento de Pessoal de Nível Superior - Brasil (CAPES), finance code 001.

\section{Author Contributions}

Julie Calmettes: data collection, data analysis, design, and draft. Roxane Peres: data collection and draft. Bruno Goncalves: data collection and draft. Clotilde Nardin: data collection, design, and draft. Elodie Meppiel: data collection, design, and draft. David Varlan: data collection and data analysis. Guillaume Turc: data collection and draft. Michael Obadia: data collection and draft. Thomas De Broucker: design, data analysis, and supervising. Mikael Mazighi: design, draft, and supervising. Aicha Lyoubi: design, data collection, data analysis, supervising, and draft.

\section{References}

1 Lodigiani C, Iapichino G, Carenzo L, Cecconi M, Ferrazzi P, Sebastian T, et al. Venous and arterial thromboembolic complications in COVID-19 patients admitted to an academic hospital in Milan, Italy. Thromb Res. 2020 Jul 1;191:9-14.

2 Mao L, Jin $\mathrm{H}$, Wang M, Hu Y, Chen S, He Q, et al. Neurologic manifestations of hospitalized patients with coronavirus disease 2019 in Wuhan, China. JAMA Neurol. 2020 01;77(6):683-90.

3 Beyrouti R, Adams ME, Benjamin L, Cohen H, Farmer SF, Goh YY, et al. Characteristics of ischaemic stroke associated with COVID-19. J Neurol Neurosurg Psychiatry. 2020;91(8):889-91.

4 Meppiel E, Peiffer-Smadja N, Maury A, Bekri I, Delorme C, Desestret V, et al. Neurological manifestations associated with COVID-19: a multicentric registry. Clin Microbiol Infect. 2020 Nov 13. Available from: https://www.ncbi.nlm.nih.gov/ pmc/articles/PMC7661948/.
5 Oxley TJ, Mocco J, Majidi S, Kellner CP, Shoirah H, Singh IP, et al. Large-vessel stroke as a presenting feature of covid-19 in the young. N Engl J Med. 2020 Apr 28. Available from: https://www.ncbi.nlm.nih. gov/pmc/articles/PMC7207073/.N Engl J Med [Internet]

6 Yaghi S, Ishida K, Torres J, Mac Grory B, Raz E, Humbert K, et al. SARS-CoV-2 and stroke in a New York healthcare system. Stroke. 2020;51(7):2002-11.

7 Klok FA, Kruip MJHA, van der Meer NJM, Arbous MS, Gommers DAMPJ, Kant KM, et al. Incidence of thrombotic complications in critically ill ICU patients with COVID-19. Thromb Res. 2020;191:145-7.

8 Zhou F, Yu T, Du R, Fan G, Liu Y, Liu Z, et al. Clinical course and risk factors for mortality of adult inpatients with COVID-19 in Wuhan, China: a retrospective cohort study. Lancet. 2020 28;395(10229):1054-62.
9 Brott T, Adams HP, Olinger CP, Marler JR, Barsan WG, Biller J, et al. Measurements of acute cerebral infarction: a clinical examination scale. Stroke. 1989 Jul;20(7):864-70.

10 Adams HP, Bendixen BH, Kappelle LJ, Biller J, Love BB, Gordon DL, et al. Classification of subtype of acute ischemic stroke. Definitions for use in a multicenter clinical trial. TOAST. trial of Org 10172 in acute stroke treatment. Stroke. 1993 Jan;24(1): 35-41.

11 Van Swieten JC, Koudstaal PJ, Visser MC, Schouten HJ, van Gijn J. Interobserver agreement for the assessment of handicap in stroke patients. Stroke. 1988 May;19(5): 604-7.

12 Jillella DV, Janocko NJ, Nahab F, Benameur K, Greene JG, Wright WL, et al. Ischemic stroke in COVID-19: An urgent need for early identification and management. PLOS ONE. 2020 Sep 18;15(9):e0239443 
13 Gabet A, Grimaud O, de Peretti C, Béjot Y, Olié V. Determinants of case fatality after hospitalization for stroke in France 2010 to 2015. Stroke. 2019;50(2):305-12.

14 Lecoffre C, de Peretti C, Gabet A, Grimaud O, Woimant F, Giroud M, et al. National trends in patients hospitalized for stroke and stroke mortality in France, 2008 to 2014. Stroke. 2017;48(11):2939-45.

15 Varga Z, Flammer AJ, Steiger P, Haberecker M, Andermatt R, Zinkernagel AS, et al. Endothelial cell infection and endotheliitis in COVID-19. The Lancet. 202;395(10234): $1417-8$.
16 Monteil V, Kwon H, Prado P, Hagelkrüys A, Wimmer RA, Stahl M, et al. Inhibition of SARS-CoV-2 infections in engineered human tissues using clinical-grade soluble human ACE2. Cell. 2020;181(4):905-e7.

17 Warren-Gash C, Smeeth L, Hayward AC. Influenza as a trigger for acute myocardial infarction or death from cardiovascular disease: a systematic review. Lancet Infect Dis. 2009 Oct;9(10):601-10.
18 Bova IY, Bornstein NM, Korczyn AD. Acute infection as a risk factor for ischemic stroke. Stroke. 1996 Dec;27(12):2204-6.

19 Majidi S, Fifi JT, Ladner TR, Lara-Reyna J, Yaeger KA, Yim B, et al. Emergent large vessel occlusion stroke during New York City's COVID-19 outbreak: clinical characteristics and paraclinical findings. Stroke. 2020 Sep;51(9):2656-63.

20 John S, Kesav P, Mifsud VA, PiechowskiJozwiak B, Dibu J, Bayrlee A, et al. Characteristics of large-vessel occlusion associated with COVID-19 and ischemic stroke. Am J Neuroradiol. 2020 Aug 27. Available from: http: //www.ajnr.org/lookup/doi/ 10.3174/ajnr.A6799. 